\title{
Replacement of dietary saturated with unsaturated fatty acid has beneficial effects in lowering plasma E-selectin and P-selectin concentrations - Results from the RISSCI-1 study
}

\author{
G. Wong ${ }^{1}$, A. Koutsos ${ }^{1}$, R. Antoni ${ }^{2}$, E. Ozen ${ }^{1}$, L. Sellem ${ }^{1}$, H. Ayyad ${ }^{2}$, B. Fielding ${ }^{2}$, \\ M.D. Robertson ${ }^{2}$, K.G. Jackson ${ }^{1}$, B.A. Griffin ${ }^{2}$ and J.A. Lovegrove ${ }^{1}$ \\ ${ }^{1}$ Hugh Sinclair Unit of Human Nutrition, University of Reading, Whiteknights, Reading, UK and \\ ${ }^{2}$ Department of Nutritional Sciences, Faculty of Health and Medical Sciences, University of Surrey, Guildford, UK
}

E-selectin and P-selectin are the major cell adhesion molecules expressed by endothelium cells. Higher circulating concentrations of E-selectin and P-selectin have been associated with the development of atherosclerotic plaque and an increase in cardiovascular disease (CVD) risk $^{(1)}$. Reductions in E-selectin has previously been demonstrated after dietary substitution of saturated fatty acids (SFA) with monounsaturated fatty acids ${ }^{(2)}$. In vitro, replacement of SFA with unsaturated fatty acids (UFA) was found to decrease platelet sensitivity to a collagen receptor (Glycoprotein VI) selective agonist in the RISSCI-1 (Reading, Imperial, Surrey Saturated Fat Cholesterol Intervention) study ${ }^{(3)}$. The aim of this study was to determine whether the replacement of dietary SFA with UFA was also associated with differences in circulating cell adhesion molecule concentrations (intercellular adhesion molecule-1 (ICAM-1), vascular adhesion molecule-1 (VCAM-1), E-selectin and P-selectin) in men from the RISSCI-1 study.

Healthy men $(\mathrm{n}=107)$, aged $30-65$ y who participated in the RISSCI-1 study (ClinicalTrials.gov Identifier NCT03270527), consumed a high-SFA diet (33\% total energy (TE) of total fat: SFA 18\% TE and UFA 15\% TE) for 4 weeks followed by a low-SFA diet (34\% TE of total fat: SFA $\leq 10 \%$ TE and UFA 24\% TE) for 4 weeks by the exchange of cooking oil, dairy, spreads and snacks high in SFA with those high in UFA. Concentrations of ICAM-1, VCAM-1, E-selectin and P-selectin were measured with the R\&D Systems Human Adhesion Molecule Multiplex kit at baseline (week 0), at the end of a high-SFA diet (week 4) and at the end of a low-SFA diet (week 8). Wilcoxon signed-rank test was performed to determine differences in cell adhesion molecule concentrations from baseline to a high-SFA diet and then a low-SFA diet.

Relative to baseline, there was a $0.4 \%$ increase in plasma E-selectin during the high-SFA diet which decreased by $5.3 \%$ following the low-SFA diet $(\mathrm{P}=0.008)$. Similarly, the changes in plasma $\mathrm{P}$-selectin from baseline was $2.6 \%$ greater in response to the high-SFA diet which was found to be reduced by $4.3 \%$ following the low-SFA diet $(\mathrm{P}=0.001)$. No significant changes were found in ICAM-1 and VCAM-1 concentrations following the high and low-SFA diets.

These findings provide evidence to suggest that the replacement of dietary SFA with UFA, in line with UK public health recommendations, may have a favourable effect on CVD risk by reducing the concentration of E-selectin and P-selectin.

\section{Acknowledgements}

RISSCI Study was funded by the BBSRC UK (reference: BB/P010245/1).

\section{References}

1. Galkina E and Ley K (2007) Arterioscler Thromb Vasc Biol 27(11), 2292-2301.

2. Vafeiadou K, Weech M, Altowaijri H, et al. (2015) Am J Clin Nutr 102, 40-8.

3. Wong G, Kriek N, Koutsos A, et al. (2021) 89th EAS Congress 137. 